Supporting information to "A modified interaction-strength interpolation method as an important step towards self-consistent calculations."

Szymon Śmiga $^{a}$, Lucian A. Constantin ${ }^{b}$

a) Institute of Physics, Faculty of Physics, Astronomy and Informatics,

Nicolaus Copernicus University, Grudziadzka 5, 87-100 Toruń, Poland

b) Center for Biomolecular Nanotechnologies @UNILE, Istituto Italiano di Tecnologia (IIT),

Via Barsanti, 73010 Arnesano (LE), Italy

May 28, 2020

\title{
Contents
}

1 Correlation energies

2 Atomization energies

3 Reaction energies

4 Vertical ionization potentials

5 Binding energies

6 GL2 correlation energies

7 Binding energies on top of HF orbitals 


\section{Correlation energies}

TABLE S1: Correlation energies (in Hartree) corresponding to various methods. The last lines report the mean absolute error (MAE) and mean absolute relative error (MARE) calculated with respect CCSD(T) data. All calculation have been performed with fully uncontacted cc-pVTZ basis set. ${ }^{a}$ ) with TPSS-GL2

\begin{tabular}{|c|c|c|c|c|c|c|c|c|}
\hline & \multirow[b]{2}{*}{$\operatorname{CCSD}(\mathrm{T})$} & \multirow[b]{2}{*}{ MP2 } & \multirow[b]{2}{*}{ OEP2-sc } & \multicolumn{5}{|c|}{$@ O E P x$} \\
\hline & & & & GL2 & ISI & mISI & $\mathrm{mISI}^{a}$ & ACSC \\
\hline $\mathrm{He}$ & -0.039660 & 0.033722 & -0.035329 & -0.044363 & -0.036901 & -0.037880 & -0.040245 & -0.037672 \\
\hline $\mathrm{Li}$ & -0.037664 & 0.032766 & -0.033113 & -0.039994 & -0.036625 & -0.037035 & -0.049778 & -0.047631 \\
\hline $\mathrm{Be}$ & -0.086245 & 0.066051 & -0.070758 & -0.113802 & -0.093827 & -0.093499 & -0.092874 & -0.079241 \\
\hline B & -0.109096 & .084970 & -0.087452 & -0.134885 & -0.115990 & -0.115052 & -0.116710 & -0.106086 \\
\hline $\mathrm{C}$ & -0.135090 & -0.111021 & -0.113715 & -0.161406 & -0.142634 & -0.141143 & -0.150590 & -0.138592 \\
\hline $\mathrm{N}$ & -0.162907 & 143243 & 89 & -0.191277 & -0.172266 & -0.170403 & -0.191004 & -0.175988 \\
\hline $\mathrm{O}$ & -0.214710 & -0.194242 & -0.197836 & -0.254493 & -0.227236 & -0.223866 & -0.238568 & -0.227975 \\
\hline $\mathrm{F}$ & 39 & 254910 & 624 & -0.326869 & -0.290263 & -0.285027 & -0.295228 & -0.283542 \\
\hline $\mathrm{Ne}$ & -0.330714 & -0.323416 & -0.352746 & -0.405145 & -0.358920 & -0.351796 & -0.358131 & -0.341914 \\
\hline $\mathrm{BeH}$ & 363 & 070275 & & -0.097580 & -0.086060 & -0.086295 & 01597 & -0.095427 \\
\hline $\mathrm{OH}$ & 866 & -0.251585 & 501 & -0.340946 & -0.293686 & -0.287072 & -0.287883 & -0.274333 \\
\hline $\mathrm{NH}_{2}$ & 278 & 233404 & -0.2 & -0.327428 & -0.276327 & -0.269931 & -0.281918 & -0.266560 \\
\hline $\mathrm{NH}$ & -0.209239 & -0.184706 & -0.190263 & -0.254288 & -0.221721 & -0.218033 & -0.233549 & -0.220886 \\
\hline $\mathrm{NO}$ & 98 & 963 & 65 & -0.723276 & -0.598771 & -0.578557 & 251 & -0.483107 \\
\hline $\mathrm{PH}_{2}$ & & & & -0.422856 & -0.399211 & -0.395922 & & 3883 \\
\hline $\mathrm{O}_{2}$ & -0.560216 & -0.538007 & -0.5 & -0.770850 & -0.644323 & -0.623286 & -0.553919 & -0.521423 \\
\hline $\mathrm{S}_{2}$ & & & & & & 57520 & 655 & 0837 \\
\hline $\mathrm{SiH}_{2}$ & -0.288381 & -0.261218 & -0.272959 & -0.347422 & -0.330311 & -0.328249 & -0.561487 & -0.530983 \\
\hline $\mathrm{C}_{2} \mathrm{H}_{5}$ & & & & & -0.501556 & -0.489244 & 714 & -0.516717 \\
\hline $\mathrm{CH}_{2}$ & -0.216482 & -0.184017 & -0.167273 & -0.278421 & -0.230092 & -0.225144 & -0.235491 & -0.216760 \\
\hline $\mathrm{CH}_{3}$ & & & & & -0.242062 & -0.237557 & 475 & -0.255391 \\
\hline $\mathrm{CN}$ & -0.4 & -0.368123 & -0 & -0.541471 & -0.454037 & -0.441432 & -0.416596 & -0.384800 \\
\hline $\mathrm{COH}$ & & & & -0.670735 & & -0.538997 & 3263 & -0.469853 \\
\hline $\mathrm{CH}_{4}$ & -0.27 & -0.244444 & 1094 & -0.348835 & -0.286640 & -0.280100 & -0.316937 & -0.296330 \\
\hline $\mathrm{LiF}$ & 9001 & -0.368097 & 3314 & -0.487876 & -0.418808 & -0.409232 & 86879 & -0.366502 \\
\hline $\mathrm{Li}_{2}$ & 7293 & -0.087716 & 9084 & -0.137012 & -0.116623 & -0.117194 & -0.133762 & -0.114303 \\
\hline $\mathrm{F}_{2}$ & 1028 & 630457 & 4311 & -0.875886 & -0.742294 & -0.719318 & -0.635842 & -0.601346 \\
\hline $\mathrm{CO}$ & -0.476203 & -0.452759 & -0.367822 & -0.659650 & -0.544370 & -0.526297 & -0.472407 & -0.438719 \\
\hline $\mathrm{CH}_{2}$ & -0.216482 & -0.184017 & -0. & -0.278421 & -0.230092 & -0.225144 & -0.235491 & -0.216760 \\
\hline $\mathrm{H}_{2}$ & -0.039681 & -0.031980 & -0.032188 & -0.047537 & -0.035415 & -0.037274 & -0.040603 & -0.040486 \\
\hline $\mathrm{CS}$ & -0.558780 & -0.519899 & 6076 & -0.781015 & -0.711328 & -0.699825 & -0.852923 & -0.781827 \\
\hline $\mathrm{LiH}$ & -0.073802 & -0.060677 & -0.061393 & -0.086328 & -0.073108 & -0.073835 & -0.083349 & -0.076529 \\
\hline $\mathrm{N}_{2}$ & -0.491774 & -0.471751 & -0.496969 & -0.708486 & -0.572963 & -0.551501 & -0.485747 & -0.446633 \\
\hline $\mathrm{P}_{2}$ & -0.660820 & -0.621909 & -0.653345 & -0.910610 & -0.852628 & -0.843207 & -1.211761 & -1.112793 \\
\hline $\mathrm{NaCl}$ & -0.579755 & -0.553435 & -0.569281 & -0.729401 & -0.690595 & -0.685110 & -1.101806 & -1.034700 \\
\hline $\mathrm{H}_{2} \mathrm{O}$ & -0.329409 & -0.314495 & -0.323122 & -0.433049 & -0.361010 & -0.350379 & -0.340403 & -0.321116 \\
\hline & & & & & & 34.85 & & 73.58 \\
\hline & MARE [\%] & 10.47 & 8.73 & 26.57 & 10.35 & 8.28 & 21.44 & 16.91 \\
\hline
\end{tabular}




\section{Atomization energies}

TABLE S2: Atomization energies (in kcal/mol) of 27 molecules from G2 set corresponding to various methods. The last lines report the mean absolute error (MAE) and mean absolute relative error (MARE) calculated with respect CCSD(T) data. All calculation have been performed with fully uncontacted cc-pVTZ basis set. ${ }^{a}$ ) with TPSS-GL2

\begin{tabular}{|c|c|c|c|c|c|c|c|c|}
\hline & \multirow[b]{2}{*}{$\operatorname{CCSD}(\mathrm{T})$} & \multirow[b]{2}{*}{ MP2 } & \multirow[b]{2}{*}{ OEP2-sc } & \multicolumn{5}{|c|}{ @OEPx } \\
\hline & & & & GL2 & ISI & mISI & $\mathrm{mISI}^{a}$ & $\mathrm{ACSC}$ \\
\hline $\mathrm{BeH}$ & -49.15 & -52.98 & -53.01 & -39.92 & -42.19 & -42.17 & -52.6 & -57.28 \\
\hline $\mathrm{OH}$ & -103.44 & -103.55 & -104.22 & -121.36 & -105.77 & -103.37 & -95.08 & -93.23 \\
\hline $\mathrm{NH}_{2}$ & -176.28 & -173.01 & -174.07 & -200.95 & -174.72 & -171.15 & -166.6 & -166.39 \\
\hline $\mathrm{NH}$ & -79.69 & -76.64 & -77.45 & -89.6 & -78.05 & -76.54 & -73.78 & -75.26 \\
\hline $\mathrm{NO}$ & -144.92 & -152.57 & -162.43 & -224.93 & -175.84 & -166.44 & -105.19 & -100.46 \\
\hline $\mathrm{PH}_{2}$ & -149.07 & -142.01 & -143.65 & -159.39 & -147.72 & -146.1 & -153.92 & -147.3 \\
\hline $\mathrm{O}_{2}$ & -115.11 & -126.86 & -135.52 & -195.27 & -150.09 & -141.11 & -79.13 & -72.04 \\
\hline $\mathrm{S}_{2}$ & -94.41 & -100.09 & -105.18 & -142.12 & -131.3 & -129.07 & -103.31 & -85.56 \\
\hline $\mathrm{SiH}_{2}$ & -129.98 & -126.63 & -127.48 & -132.36 & -123.82 & -122.8 & -137.63 & -134.82 \\
\hline $\mathrm{C}_{2} \mathrm{H} 5$ & -593.95 & -593.7 & -596 & -641.03 & -586.57 & -578.9 & -609.65 & -601.49 \\
\hline $\mathrm{CH}_{2}$ & -187.46 & -187.02 & -187.52 & -195.64 & -182.36 & -180.91 & -195.93 & -194.39 \\
\hline $\mathrm{CH}_{3}$ & -303 & -300.77 & -301.33 & -321.41 & -295.41 & -292.43 & -309.07 & -306.51 \\
\hline $\mathrm{CN}$ & -171.62 & -161.44 & -194.38 & -207.01 & -175.86 & -170.05 & -135.61 & -132.61 \\
\hline $\mathrm{COH}$ & -271.53 & -282.15 & -288 & -340.18 & -294.35 & -286.03 & -248.89 & -242.1 \\
\hline $\mathrm{CH}_{4}$ & -414.67 & -411.71 & -412.49 & -444.7 & -405.27 & -400.65 & -419.56 & -414.15 \\
\hline $\mathrm{LiF}$ & -134.05 & -140.47 & -142.08 & -166.01 & -147.76 & -144.77 & -116.35 & -112.24 \\
\hline $\mathrm{Li}_{2}$ & -24 & -17.87 & -17.97 & -39.56 & -31 & -30.84 & -25.25 & -15.73 \\
\hline $\mathrm{F}_{2}$ & -35.38 & -40.96 & -47.34 & -101.76 & -63.87 & -56.03 & 9.16 & 16.14 \\
\hline $\mathrm{CO}$ & -253.5 & -266.73 & -271.54 & -325.78 & -282.32 & -274.03 & -225.06 & -218.1 \\
\hline $\mathrm{CH}_{2}$ & -177.08 & -171.81 & -172.29 & -198.61 & -173.98 & -171.08 & -172.5 & -168.28 \\
\hline $\mathrm{H}_{2}$ & -108.64 & -103.8 & -103.85 & -113.57 & -99.87 & -100.31 & -103.26 & -103.19 \\
\hline CS & -164.1 & -172.12 & -178.58 & -241.72 & -220.75 & -215.95 & -151.98 & -136.4 \\
\hline $\mathrm{LiH}$ & -56.86 & -51.69 & -51.75 & -63.2 & -53.97 & -53.81 & -52.21 & -49.28 \\
\hline $\mathrm{N}_{2}$ & -218.75 & -230.86 & -235.74 & -317.06 & -255.88 & -244.75 & -177.64 & -171.94 \\
\hline $\mathrm{P}_{2}$ & -104.75 & -107.94 & -112.76 & -181.65 & -163.77 & -160.21 & -103.6 & -81.6 \\
\hline $\mathrm{NaCl}$ & -93.3 & -96.27 & -96.1 & -111.04 & -106.48 & -105.61 & -100.02 & -92.66 \\
\hline \multirow[t]{3}{*}{$\mathrm{H}_{2} \mathrm{O}$} & -226.28 & -229.77 & -230.7 & -265.92 & -231.73 & -226.45 & -211.82 & -206.37 \\
\hline & MAE & 5.51 & 8.01 & 37.75 & 16.43 & 14.51 & 14.83 & 17.76 \\
\hline & MARE[\%] & 5.19 & 7.32 & 31.59 & 15.03 & 13.00 & 13.22 & 17.16 \\
\hline
\end{tabular}

TABLE S3: Atomization energies (in kcal/mol) of AE6 set corresponding to various methods. The last lines report the mean absolute error (MAE) and mean absolute relative error (MARE) calculated with respect CCSD(T) data. All calculation have been performed with fully uncontacted cc-pVTZ basis set. ${ }^{a}$ ) with TPSS-GL2

\begin{tabular}{|c|c|c|c|c|c|c|c|c|}
\hline & \multirow[b]{2}{*}{$\operatorname{CCSD}(\mathrm{T})$} & \multirow[b]{2}{*}{ MP2 } & \multirow[b]{2}{*}{ OEP2-sc } & \multicolumn{5}{|c|}{ @OEPx } \\
\hline & & & & GL2 & ISI & mISI & $\mathrm{mISI}^{a}$ & ACSC \\
\hline $\mathrm{SiH}_{4}$ & 318.8 & 310.2 & 311.2 & 332.5 & 324.6 & 323.7 & 333.2 & 318 \\
\hline $\mathrm{SiO}$ & 182 & 194.7 & 201 & 255.1 & 230.1 & 225 & 149.9 & 142.5 \\
\hline $\mathrm{S}_{2}$ & 94.3 & 100 & 104.9 & 140.6 & 130 & 127.9 & 104.9 & 86.9 \\
\hline $\mathrm{C}_{3} \mathrm{H}_{4}$ & 690.8 & 703.4 & 707.2 & 789.4 & 723.4 & 712.8 & 704.1 & 680.5 \\
\hline $\mathrm{C}_{2} \mathrm{H}_{2} \mathrm{O}_{2}$ & 618.1 & 641.6 & 651.9 & 761.8 & 671.5 & 654.1 & 586.2 & 564 \\
\hline \multirow[t]{3}{*}{$\mathrm{C}_{4} \mathrm{H}_{8}$} & -1130.5 & -1140.7 & -1145.6 & -1247.9 & -1150.7 & -1132.3 & -1181.1 & -1144.8 \\
\hline & MAE & 12.2 & 17.1 & 82.1 & 32.7 & 23.6 & 25.5 & 21 \\
\hline & MARE[\%] & 3.71 & 5.55 & 23.59 & 13.56 & 11.67 & 7.51 & 6.87 \\
\hline
\end{tabular}


TABLE S4: Atomization energies (in $\mathrm{kcal} / \mathrm{mol}$ ) of small radicals set corresponding to various methods. The last lines report the mean absolute error (MAE) and mean absolute relative error (MARE) calculated with respect CCSD(T) data. All calculation have been performed with fully uncontacted cc-pVTZ basis set. ${ }^{a}$ ) with TPSS-GL2

\begin{tabular}{|c|c|c|c|c|c|c|c|c|}
\hline & \multirow[b]{2}{*}{$\operatorname{CCSD}(\mathrm{T})$} & \multirow[b]{2}{*}{ MP2 } & \multirow[b]{2}{*}{ OEP2-sc } & \multicolumn{5}{|c|}{ @OEPx } \\
\hline & & & & GL2 & ISI & mISI & $\mathrm{mISI}^{a}$ & ACSC \\
\hline $\mathrm{BH}_{2}$ & -167.4 & -167.9 & -168.0 & -169.2 & -163.6 & -162.8 & -177.9 & -174.5 \\
\hline $\mathrm{BH}^{+}$ & 218.0 & 217.2 & 136.5 & 148.4 & 144.9 & 144.7 & 134.5 & 135.0 \\
\hline $\mathrm{BO}$ & -189.7 & -204.1 & -209.5 & -240.0 & -211.8 & -206.3 & -167.5 & -164.4 \\
\hline $\mathrm{C}_{2}^{-}$ & -210.3 & -222.3 & -229.8 & -317.3 & -250.0 & -238.6 & -184.6 & -175.9 \\
\hline $\mathrm{CF}$ & -129.6 & -134.8 & -140.2 & -165.3 & -143.5 & -139.0 & -110.2 & -107.9 \\
\hline $\mathrm{CH}$ & -82.0 & -78.4 & -78.8 & -91.1 & -82.0 & -80.7 & -80.1 & -77.7 \\
\hline $\mathrm{CN}$ & -174.0 & -161.0 & -194.4 & -206.4 & -175.3 & -169.5 & -135.3 & -132.3 \\
\hline $\mathrm{CO}^{+}$ & 66.2 & 62.1 & 45.2 & 17.9 & 48.2 & 53.9 & 103.0 & 107.3 \\
\hline $\mathrm{F}_{2}^{+}$ & 321.0 & 310.8 & 298.7 & 214.7 & 270.3 & 281.2 & 390.1 & 395.4 \\
\hline $\mathrm{FH}^{+}$ & 227.4 & 228.7 & 228.3 & 225.9 & 228.1 & 228.7 & 231.0 & 232.8 \\
\hline $\mathrm{H}_{2} \mathrm{O}^{+}$ & 60.1 & 61.0 & 60.5 & 44.7 & 57.8 & 60.2 & 65.4 & 70.5 \\
\hline $\mathrm{HCO}$ & -272.6 & -282.2 & -288.0 & -340.0 & -296.6 & -288.3 & -250.8 & -242.4 \\
\hline HNF & -151.4 & -152.5 & -159.5 & -202.1 & -167.2 & -160.1 & -124.2 & -119.3 \\
\hline $\mathrm{HOO}$ & -169.0 & -170.8 & -183.1 & -234.4 & -190.3 & -181.4 & -134.8 & -125.8 \\
\hline $\mathrm{N}_{2}^{+}$ & 136.3 & 121.4 & 109.0 & -17.7 & 76.7 & 92.4 & 208.6 & 213.6 \\
\hline $\mathrm{NH}_{2}$ & -176.4 & -173.1 & -174.1 & -200.6 & -179.6 & -176.2 & -170.4 & -166.9 \\
\hline $\mathrm{NH}^{+}$ & 228.4 & 235.3 & 234.8 & 221.3 & 228.8 & 229.9 & 241.5 & 241.0 \\
\hline $\mathrm{NO}$ & -146.0 & -152.7 & -162.4 & -225.0 & -175.9 & -166.4 & -105.3 & -100.6 \\
\hline $\mathrm{O}_{2}^{+}$ & 158.6 & 141.4 & 132.9 & 38.7 & 106.2 & 118.8 & 217.9 & 226.3 \\
\hline $\mathrm{OF}$ & -49.9 & -50.5 & -63.0 & -99.5 & -68.6 & -62.2 & -12.6 & -7.9 \\
\hline \multirow[t]{3}{*}{$\mathrm{OH}$} & -103.5 & -103.6 & -104.3 & -121.2 & -108.1 & -105.7 & -97.0 & -93.5 \\
\hline & MAE & 6.1 & 15.7 & 52.98 & 21.69 & 16.59 & 30.23 & 34.23 \\
\hline & MARE[\%] & 3.89 & 12.05 & 89.10 & 16.97 & 12.18 & 32.10 & 46.35 \\
\hline
\end{tabular}


TABLE S5: Energies (in kcal/mol) of K9 set corresponding to various methods. The last lines report the mean absolute error (MAE) and mean absolute relative error (MARE) calculated with respect $\operatorname{CCSD}(\mathrm{T})$ data. All calculation have been performed with fully uncontacted cc-pVTZ basis set. ${ }^{a}$ ) with TPSS-GL2

\begin{tabular}{lcccccccc}
\hline \hline & & & & \multicolumn{5}{c}{ @OEPx } \\
\cline { 5 - 8 } & CCSD $(\mathrm{T})$ & MP2 & OEP2-sc & GL2 & ISI & mISI & mISI $^{a}$ & ACSC \\
\hline $\mathrm{OH}+\mathrm{CH}_{4} \rightarrow \mathrm{TS}$ & 7.1 & 8.52 & 2.07 & -7.04 & 2.95 & 5.69 & 18.48 & 20.25 \\
$\mathrm{TS} \rightarrow \mathrm{CH}_{3}+\mathrm{H}_{2} \mathrm{O}$ & 18.25 & 23.74 & 17.3 & 14.05 & 18.97 & 20.49 & 24.78 & 25.79 \\
$\mathrm{H}+\mathrm{OH} \rightarrow \mathrm{TS}$ & 50.1 & 55.43 & 13.35 & 13.9 & 13.93 & 13.65 & 13.59 & 15.06 \\
$\mathrm{TS} \rightarrow \mathrm{O}+\mathrm{H}_{2}$ & 55.19 & 55.63 & 12.93 & 6.27 & 11.12 & 14.03 & 23.15 & 24.71 \\
$\mathrm{H}+\mathrm{H}_{2} \mathrm{~S} \rightarrow \mathrm{TS}$ & 4.1 & 6.77 & 5.66 & 3.09 & 4.06 & 4.16 & 5.94 & 6.5 \\
$\mathrm{TS} \rightarrow \mathrm{H}_{2}+\mathrm{SH}$ & 18.93 & 17.37 & 16.15 & 9.92 & 8.03 & 10.15 & 11.73 & 18.62 \\
& & & & & & & & \\
& $\mathrm{MAE}$ & 2.82 & 14.89 & 18.91 & 16.01 & 15.01 & 15.92 & 14.82 \\
& MARE[\%] & 22.46 & 46.45 & 75.88 & 45.52 & 37.87 & 68.32 & 68.63 \\
\hline \hline
\end{tabular}




\section{Reaction energies}

TABLE S6: Hydrogen transfer reactions energies (in kcal/mol) corresponding to various methods. The last lines report the mean absolute error (MAE) and mean absolute relative error (MARE) calculated with respect CCSD(T) data. All calculation have been performed with fully uncontacted cc-pVTZ basis set. ${ }^{a}$ ) with TPSS-GL2

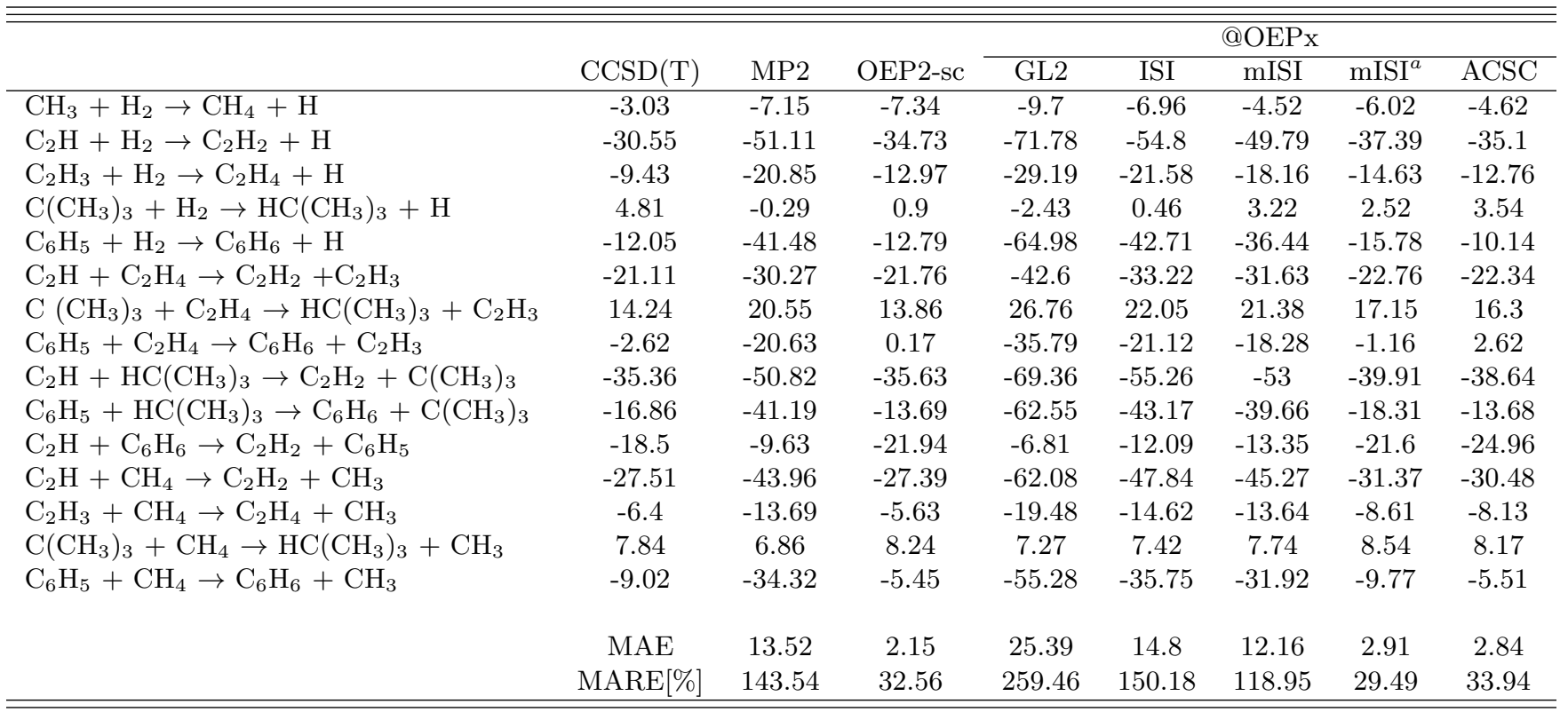

TABLE S7: Isomerization/reaction energies (in $\mathrm{kcal} / \mathrm{mol}$ ) corresponding to various methods. The last lines report the mean absolute error (MAE) and mean absolute relative error (MARE) calculated with respect CCSD(T) data. All calculation have been performed with fully uncontacted cc-pVTZ basis set. ${ }^{a}$ ) with TPSS-GL2

\begin{tabular}{|c|c|c|c|c|c|c|c|c|}
\hline & \multirow[b]{2}{*}{$\operatorname{CCSD}(\mathrm{T})$} & \multirow[b]{2}{*}{ MP2 } & \multirow[b]{2}{*}{ OEP2-sc } & \multicolumn{5}{|c|}{ @OEPx } \\
\hline & & & & GL2 & ISI & mISI & $\mathrm{mISI}^{a}$ & $\mathrm{ACSC}$ \\
\hline 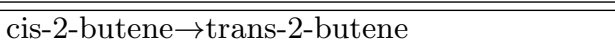 & 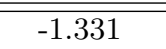 & 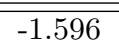 & 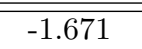 & $\begin{array}{c}-1.958 \\
\end{array}$ & -1.658 & $\overline{-1.508}$ & 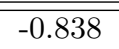 & 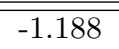 \\
\hline methylformate $\rightarrow$ acetic acid & -16.528 & -17.43 & -16.406 & -15.848 & -16.32 & -16.398 & -21.717 & -20.579 \\
\hline Propanone $\rightarrow$ Propanal & -10.543 & -10.823 & -11.012 & -9.651 & -10.339 & -10.404 & -11.573 & -11.506 \\
\hline Acetylenediol $\rightarrow$ Glioxal & 46.733 & 44.385 & 46.563 & 47.096 & 46.985 & 47.191 & 37.038 & 39.885 \\
\hline Water-methanol $1 \rightarrow$ Water-methanol 2 & -0.098 & -0.107 & -0.121 & -0.213 & -0.133 & -0.117 & 0.001 & 0.075 \\
\hline Methylenecyclopropene $\rightarrow$ Cumulene & 15.788 & 12.248 & 15.362 & 26.964 & 20.04 & 18.997 & -8.516 & -4.995 \\
\hline Methylenecyclopropene $\rightarrow$ Cyclobutadiene & -58.429 & -61.826 & -58.475 & 77.872 & 25.305 & 16.937 & -52.645 & -50.569 \\
\hline Methylenecyclopropene $\rightarrow$ Vinylacetylene & 23.81 & 23.772 & 23.798 & 35.923 & 29.722 & 28.809 & 2.495 & 6.395 \\
\hline Cumulene $\rightarrow$ Cyclobutadiene & -74.217 & -74.075 & -73.838 & 50.908 & 5.265 & -2.06 & -44.129 & -45.574 \\
\hline Cumulene $\rightarrow$ Vinylacetylene & 8.022 & 11.524 & 8.436 & 8.959 & 9.682 & 9.812 & 11.011 & 11.39 \\
\hline Cyclobutadiene $\rightarrow$ Vinylacetylene & 82.239 & 85.599 & 82.274 & -41.949 & 4.417 & 11.872 & 55.14 & 56.964 \\
\hline$\left(\mathrm{H}_{2} \mathrm{O}\right)_{6}$ book $\rightarrow\left(\mathrm{H}_{2} \mathrm{O}\right)_{6}$ book2 & -1.413 & -1.449 & -1.383 & -0.872 & -1.263 & -1.297 & -2.003 & -2.032 \\
\hline$\left(\mathrm{H}_{2} \mathrm{O}\right)_{6}$ book $\rightarrow\left(\mathrm{H}_{2} \mathrm{O}\right)_{6}$ ring & -2.138 & -2.096 & -2.762 & -4.507 & -2.963 & -2.316 & 0.291 & 0.292 \\
\hline$\left(\mathrm{H}_{2} \mathrm{O}\right)_{6}$ book $\rightarrow\left(\mathrm{H}_{2} \mathrm{O}\right)_{6}$ prism & 2.226 & 2.076 & 2.82 & 4.558 & 3.109 & 2.362 & -0.016 & -0.226 \\
\hline$\left(\mathrm{H}_{2} \mathrm{O}\right)_{6}$ book $\rightarrow\left(\mathrm{H}_{2} \mathrm{O}\right)_{6}$ cage & 1.459 & 1.4 & 1.909 & 3.211 & 2.096 & 1.565 & -0.291 & -0.416 \\
\hline$\left(\mathrm{H}_{2} \mathrm{O}\right)_{6}$ book $2 \rightarrow\left(\mathrm{H}_{2} \mathrm{O}\right)_{6}$ ring & -0.724 & -0.647 & -1.379 & -3.635 & -1.701 & -1.018 & 2.295 & 2.324 \\
\hline$\left(\mathrm{H}_{2} \mathrm{O}\right)_{6}$ book $2 \rightarrow\left(\mathrm{H}_{2} \mathrm{O}\right)_{6}$ prism & 3.639 & 3.525 & 4.203 & 5.43 & 4.372 & 3.66 & 1.988 & 1.806 \\
\hline$\left(\mathrm{H}_{2} \mathrm{O}\right)_{6}$ book $2 \rightarrow\left(\mathrm{H}_{2} \mathrm{O}\right)_{6}$ cage & 2.872 & 2.849 & 3.292 & 4.083 & 3.359 & 2.862 & 1.713 & 1.616 \\
\hline$\left(\mathrm{H}_{2} \mathrm{O}\right)_{6}$ ring $\rightarrow\left(\mathrm{H}_{2} \mathrm{O}\right)_{6}$ prism & 4.363 & 4.172 & 5.582 & 9.065 & 6.072 & 4.678 & -0.307 & -0.517 \\
\hline$\left(\mathrm{H}_{2} \mathrm{O}\right)_{6}$ ring $\rightarrow\left(\mathrm{H}_{2} \mathrm{O}\right)_{6}$ cage & 3.596 & 3.496 & 4.671 & 7.718 & 5.06 & 3.881 & -0.582 & -0.708 \\
\hline \multirow[t]{3}{*}{$\left(\mathrm{H}_{2} \mathrm{O}\right)_{6}$ prism $\rightarrow\left(\mathrm{H}_{2} \mathrm{O}\right)_{6}$ cage } & -0.767 & -0.676 & -0.911 & -1.347 & -1.013 & -0.797 & -0.275 & -0.19 \\
\hline & MAE & 0.89 & 0.39 & 20.71 & 12.48 & 10.97 & 7.16 & 6.61 \\
\hline & MARE[\%] & 7.97 & 16.59 & 96.60 & 42.74 & 23.87 & 82.39 & 84.89 \\
\hline
\end{tabular}


TABLE S8: Close-shell reaction energies (in $\mathrm{kcal} / \mathrm{mol}$ ) corresponding to various methods. The last lines report the mean absolute error (MAE) and mean absolute relative error (MARE) calculated with respect $\operatorname{CCSD}(\mathrm{T})$ data. All calculation have been performed with fully uncontacted cc-pVTZ basis set. ${ }^{a}$ ) with TPSS-GL2

\begin{tabular}{|c|c|c|c|c|c|c|c|c|}
\hline & \multirow[b]{2}{*}{$\operatorname{CCSD}(\mathrm{T})$} & \multirow[b]{2}{*}{ MP2 } & \multirow[b]{2}{*}{ OEP2-sc } & \multicolumn{5}{|c|}{ @OEPx } \\
\hline & & & & GL2 & ISI & mISI & $\mathrm{mISI}^{a}$ & ACSC \\
\hline $\mathrm{F}_{2} \mathrm{O}+\mathrm{H}_{2} \rightarrow \mathrm{F}_{2}+\mathrm{H}_{2} \mathrm{O}$ & -66.83 & -70.1 & -62.78 & -51.36 & -61.53 & -62.39 & -84.4 & -84.06 \\
\hline $\mathrm{H}_{2} \mathrm{O}_{2}+\mathrm{H}_{2} \rightarrow 2 \mathrm{H}_{2} \mathrm{O}$ & -84.27 & -88.41 & -85.31 & -73 & -81.29 & -81.78 & -100.43 & -99.96 \\
\hline $\mathrm{CO}+\mathrm{H}_{2} \rightarrow \mathrm{CH}_{2} \mathrm{O}$ & -3.55 & -4 & -3.51 & 2.81 & -0.66 & 0.76 & -18.33 & -15.88 \\
\hline $\mathrm{CO}+3 \mathrm{H}_{2} \rightarrow \mathrm{CH}_{4}+\mathrm{H}_{2} \mathrm{O}$ & -61.55 & -63.33 & -60.11 & -43.96 & -54.98 & -52.04 & -96.55 & -92.87 \\
\hline $\mathrm{N}_{2}+3 \mathrm{H}_{2} \rightarrow 2 \mathrm{NH}_{3}$ & -34.7 & -34.22 & -31.3 & -3.05 & -21.26 & -19.43 & -69.6 & -68.37 \\
\hline $\mathrm{N}_{2} \mathrm{O}+\mathrm{H}_{2} \rightarrow \mathrm{N}_{2}+\mathrm{H}_{2} \mathrm{O}$ & -79.86 & -75.13 & -65.2 & -45.43 & -66.06 & -68.82 & -91.89 & -94.06 \\
\hline $\mathrm{HNO}_{2}+3 \mathrm{H}_{2} \rightarrow 2 \mathrm{H}_{2} \mathrm{O}+\mathrm{NH}_{3}$ & -117.82 & -121.89 & -108.26 & -76.06 & -102.67 & -103.56 & -158.59 & -157.86 \\
\hline $\mathrm{C} 2 \mathrm{H}_{2}+\mathrm{H}_{2} \rightarrow \mathrm{C}_{2} \mathrm{H}_{4}$ & -49.62 & -47.55 & -47.29 & -33.33 & -42.29 & -41.8 & -64.31 & -64.49 \\
\hline $\mathrm{CH}_{2}-\mathrm{CO}+2 \mathrm{H}_{2} \rightarrow \mathrm{CH}_{2} \mathrm{O}+\mathrm{CH}_{4}$ & -42.64 & -40.51 & -37.57 & -28 & -37.12 & -36.09 & -54.95 & -55.98 \\
\hline $\mathrm{BH}_{3}+3 \mathrm{HF} \rightarrow \mathrm{BF}_{3}+3 \mathrm{H}_{2}$ & -98.1 & -99.17 & -102.65 & -99.81 & -96.09 & -98.95 & -86.38 & -91.4 \\
\hline $\mathrm{HCOOH} \rightarrow \mathrm{CO}_{2}+\mathrm{H}_{2}$ & 1.17 & -1.4 & -3.19 & -11.82 & -5.03 & -6.04 & 13.36 & 12.72 \\
\hline $\mathrm{CO}+\mathrm{H}_{2} \mathrm{O} \rightarrow \mathrm{CO}_{2}+\mathrm{H}_{2}$ & -7.67 & -11.7 & -16.35 & -17.36 & -12.03 & -12.05 & -8.49 & -7.72 \\
\hline $\mathrm{C}_{2} \mathrm{H}_{2}+\mathrm{HF} \rightarrow \mathrm{CH}_{2} \mathrm{CHF}$ & -28.48 & -25.51 & -27.47 & -13.37 & -21.32 & -21.43 & -40.56 & -39.42 \\
\hline $\mathrm{HCN}+\mathrm{H}_{2} \mathrm{O} \rightarrow \mathrm{CO}+\mathrm{NH}_{3}$ & -13.37 & -10.46 & -11.27 & -0.05 & -7.55 & -8.88 & -14.89 & -17.11 \\
\hline $\mathrm{HCN}+\mathrm{H}_{2} \mathrm{O} \rightarrow \mathrm{HCONH}_{2}$ & -21.73 & -21.12 & -23.89 & -3.57 & -13.72 & -14.12 & -40.07 & -40.11 \\
\hline $\mathrm{HCONH}_{2}+\mathrm{H}_{2} \mathrm{O} \rightarrow \mathrm{HCOOH}+\mathrm{NH}_{3}$ & -0.49 & 0.35 & -0.54 & -2.02 & -0.83 & -0.77 & 3.33 & 2.56 \\
\hline $\mathrm{HCN}+\mathrm{NH}_{3} \rightarrow \mathrm{N}_{2}+\mathrm{CH}_{4}$ & -40.22 & -39.57 & -40.07 & -40.97 & -41.27 & -41.49 & -41.83 & -41.61 \\
\hline $\mathrm{CO}+\mathrm{CH}_{4} \rightarrow \mathrm{CH}_{3} \mathrm{CHO}$ & 4.56 & 3.3 & 2.62 & 7.41 & 6.54 & 7.74 & -11.52 & -7.5 \\
\hline $\mathrm{N}_{2}+\mathrm{F}_{2} \rightarrow$ trans $-\mathrm{N}_{2} \mathrm{~F}_{2}$ & 17.32 & 18.62 & 11.03 & 20.87 & 19.96 & 21.35 & 10.35 & 12.14 \\
\hline $\mathrm{N}_{2}+\mathrm{F}_{2} \rightarrow$ cis- $\mathrm{N}_{2} \mathrm{~F}_{2}$ & 18.83 & 19.51 & 16.19 & 29.07 & 24.62 & 25.33 & 8.61 & 10.21 \\
\hline $2 \mathrm{BH}_{3} \rightarrow \mathrm{B}_{2} \mathrm{H}_{6}$ & -43.09 & -44.58 & -46.07 & -57.72 & -48.1 & -45.68 & -51.06 & -46.96 \\
\hline $\mathrm{CH}_{3} \mathrm{ONO} \rightarrow \mathrm{CH}_{3} \mathrm{NO}_{2}$ & -0.89 & -5.61 & -1.7 & -1.85 & -2.11 & -2.22 & -8.16 & -7.29 \\
\hline $\mathrm{CH}_{2}-\mathrm{C} \rightarrow \mathrm{C}_{2} \mathrm{H}_{2}$ & -44.28 & -51.94 & -49.9 & -59.35 & -51.99 & -50.74 & -45.45 & -44.31 \\
\hline Allene $\rightarrow$ propyne & -1.1 & -4.43 & -3.43 & -7.61 & -5.42 & -5.16 & -3.36 & -2.86 \\
\hline Cyclopropene $\rightarrow$ propyne & -23.38 & -23.68 & -23.99 & -28.09 & -26.72 & -26.64 & -10.5 & -13.49 \\
\hline Oxirane $\rightarrow \mathrm{CH} 3 \mathrm{CHO}$ & -26.73 & -25.75 & -26.63 & -27.36 & -27.74 & -27.95 & -16.78 & -19.52 \\
\hline Vinlyalcohol $\rightarrow \mathrm{CH}_{3} \mathrm{CHO}$ & -9.79 & -10 & -10.8 & -11.8 & -11.11 & -11.18 & -9.38 & -9.01 \\
\hline Cyclobutene $\rightarrow 1,3$-butadiene & -11.5 & -9.41 & -9.81 & -11.44 & -11.72 & -11.95 & 3.4 & 1.5 \\
\hline $2 \mathrm{NH}_{3} \rightarrow\left(\mathrm{NH}_{3}\right)_{2}$ & -3.84 & -4 & -4.25 & -5.14 & -4.58 & -3.91 & -2.99 & -3.06 \\
\hline $2 \mathrm{H}_{2} \mathrm{O} \rightarrow\left(\mathrm{H}_{2} \mathrm{O}\right)_{2}$ & -5.67 & -5.84 & -6.26 & -6.84 & -6.3 & -5.67 & -4.83 & -4.93 \\
\hline \multirow[t]{3}{*}{$2 \mathrm{HF} \rightarrow(\mathrm{HF})_{2}$} & -4.19 & -4.22 & -4.81 & -5.41 & -4.77 & -4.14 & -3.03 & -3.02 \\
\hline & MAE & 2.04 & 2.98 & 10.57 & 4.66 & 4.63 & 11.4 & 10.32 \\
\hline & MARE[\%] & 46.79 & 34.53 & 106.30 & 54.47 & 57.34 & 144.77 & 125.72 \\
\hline
\end{tabular}


TABLE S9: Open-shell reaction energies (in $\mathrm{kcal} / \mathrm{mol}$ ) corresponding to various methods. The last lines report the mean absolute error (MAE) and mean absolute relative error (MARE) calculated with respect $\operatorname{CCSD}(\mathrm{T})$ data. All calculation have been performed with fully uncontacted cc-pVTZ basis set. ${ }^{a}$ ) with TPSS-GL2

\begin{tabular}{|c|c|c|c|c|c|c|c|c|}
\hline & \multirow[b]{2}{*}{$\operatorname{CCSD}(\mathrm{T})$} & \multirow[b]{2}{*}{ MP2 } & \multirow[b]{2}{*}{ OEP2-sc } & \multicolumn{5}{|c|}{$@ \mathrm{OEPx}$} \\
\hline & & & & GL2 & ISI & mISI & $\mathrm{mISI}^{a}$ & ACSC \\
\hline $\mathrm{HCl}+\mathrm{H} \rightarrow \mathrm{Cl}+\mathrm{H}_{2}$ & 4.13 & -1.17 & -1.17 & -6.1 & -8.82 & -6.75 & -5.26 & 1.46 \\
\hline $\mathrm{H}_{2} \mathrm{O}+\mathrm{F}_{2} \rightarrow 2 \mathrm{HF}+\mathrm{O}$ & 13.37 & 12.48 & 6.48 & -42.22 & -8.27 & -1.4 & 49.25 & 54.13 \\
\hline $\mathrm{CH}_{4}+\mathrm{OH} \rightarrow \mathrm{CH}_{3}+\mathrm{H}_{2} \mathrm{O}$ & 11.19 & 15.28 & 15.31 & 21.27 & 16.1 & 14.86 & 6.26 & 5.5 \\
\hline $\mathrm{CH}_{3}+\mathrm{Cl}_{2} \rightarrow \mathrm{CH}_{3} \mathrm{Cl}+\mathrm{Cl}$ & 31.32 & 31.85 & 30.75 & 23.28 & 31.5 & 31.39 & 39.76 & 37.69 \\
\hline $\mathrm{S}+2 \mathrm{HCl} \rightarrow \mathrm{H}_{2} \mathrm{~S}+\mathrm{Cl}_{2}$ & 22.54 & 23.43 & 26.05 & 49.22 & 42.3 & 40.84 & 24.01 & 13.42 \\
\hline $\mathrm{N}+\mathrm{O}_{2} \rightarrow \mathrm{NO}+\mathrm{O}$ & 29.8 & 25.71 & 26.91 & 29.66 & 25.75 & 25.33 & 26.05 & 28.42 \\
\hline $4 \mathrm{HCl}+\mathrm{O}_{2} \rightarrow 2 \mathrm{H}_{2} \mathrm{O}+2 \mathrm{Cl}_{2}$ & 25.73 & 28.07 & 25.25 & 32.55 & 24.54 & 24.47 & 15.28 & 19.12 \\
\hline $2 \mathrm{NO} \rightarrow \mathrm{N}_{2}+\mathrm{O}_{2}$ & 44.03 & 52.59 & 46.4 & 62.47 & 54.29 & 52.99 & 46.39 & 43.05 \\
\hline $2 \mathrm{H}_{2} \mathrm{O}_{2} \rightarrow 2 \mathrm{H}_{2} \mathrm{O}+\mathrm{O}_{2}$ & 48.36 & 51.74 & 52.44 & 36.69 & 49.01 & 52.47 & 62.86 & 65.6 \\
\hline $\mathrm{Cl}_{2}+\mathrm{H} \rightarrow \mathrm{HCl}+\mathrm{Cl}$ & 51.36 & 47.33 & 45.3 & 32.29 & 35.7 & 36.57 & 56.1 & 59.07 \\
\hline $2 \mathrm{SO}_{2}+\mathrm{O}_{2} \rightarrow 2 \mathrm{SO}_{3}$ & 35.68 & 44.58 & 42.25 & 26.62 & 31.9 & 31.95 & 65.02 & 59.18 \\
\hline $\mathrm{H}_{2} \mathrm{~S}+\mathrm{F}_{2} \rightarrow \mathrm{S}+2 \mathrm{HF}$ & 61.25 & 66.52 & 60.81 & 22.48 & 36.75 & 40.55 & 72.44 & 86.28 \\
\hline $\mathrm{NO}+\mathrm{N} \rightarrow \mathrm{O}+\mathrm{N}_{2}$ & 73.83 & 78.29 & 73.31 & 92.13 & 80.04 & 78.31 & 72.45 & 71.48 \\
\hline $2 \mathrm{SiH}_{3} \rightarrow \mathrm{Si}_{2} \mathrm{H}_{6}$ & 76.97 & 77.02 & 77.61 & 90.38 & 86.03 & 85.11 & 87.9 & 77.18 \\
\hline $\mathrm{CH}_{3}+\mathrm{SH} \rightarrow \mathrm{CH}_{3} \mathrm{SH}$ & 76.72 & 81.14 & 82.15 & 101.73 & 100.37 & 98.36 & 87.69 & 73.49 \\
\hline $\mathrm{CS}+\mathrm{O} \rightarrow \mathrm{CO}+\mathrm{S}$ & 89.4 & 94.62 & 92.98 & 84.05 & 61.57 & 58.08 & 73.08 & 81.7 \\
\hline $\mathrm{CH}_{3}+\mathrm{Cl} \rightarrow \mathrm{CH}_{3} \mathrm{Cl}$ & 84.47 & 89.49 & 90.46 & 110.72 & 110.61 & 108.73 & 95.53 & 80.34 \\
\hline $\mathrm{NH}+\mathrm{H} \rightarrow \mathrm{NH}_{2}$ & 96.59 & 96.37 & 96.62 & 111.34 & 99.71 & 98.01 & 94.5 & 91.13 \\
\hline $\mathrm{Si}+2 \mathrm{H}_{2} \rightarrow \mathrm{SiH}_{4}$ & 101.49 & 102.58 & 103.41 & 105.31 & 112.11 & 108.57 & 119.99 & 111.48 \\
\hline $2 \mathrm{C}_{2} \mathrm{H}_{4}+\mathrm{O}_{2} \rightarrow 2 \mathrm{CH}_{3} \mathrm{CHO}$ & 104.35 & 110.31 & 109.71 & 105.1 & 102.52 & 100.69 & 116.47 & 111.97 \\
\hline $\mathrm{NH}_{2}+\mathrm{H} \rightarrow \mathrm{NH}_{3}$ & 113.4 & 115.24 & 115.22 & 129.55 & 116.75 & 114.87 & 113.58 & 108.54 \\
\hline $2 \mathrm{H}_{2}+\mathrm{O}_{2} \rightarrow 2 \mathrm{H}_{2} \mathrm{O}$ & 120.18 & 125.07 & 118.19 & 109.32 & 113.58 & 111.1 & 138 & 134.33 \\
\hline $\mathrm{CO}_{2}+\mathrm{C} \rightarrow 2 \mathrm{CO}$ & 128.17 & 129.06 & 128.34 & 156.12 & 138.46 & 135.88 & 108 & 107.2 \\
\hline $\mathrm{N}_{2} \mathrm{H}_{4}+\mathrm{O}_{2} \rightarrow \mathrm{N}_{2}+2 \mathrm{H}_{2} \mathrm{O}$ & 132.86 & 139.43 & 134.48 & 151.22 & 139.48 & 138.47 & 119.64 & 116.77 \\
\hline $4 \mathrm{NH}_{3}+5 \mathrm{O}_{2} \rightarrow 4 \mathrm{NO}+6 \mathrm{H}_{2} \mathrm{O}$ & 203.1 & 201.58 & 199.16 & 196.92 & 189.65 & 188.46 & 182 & 180.15 \\
\hline \multirow[t]{3}{*}{$2 \mathrm{NH}_{3}+2 \mathrm{NO}+\mathrm{O} \rightarrow 2 \mathrm{~N}_{2}+3 \mathrm{H}_{2} \mathrm{O}$} & 247.16 & 269.4 & 260.13 & 321.04 & 278.44 & 270.76 & 223.35 & 212.2 \\
\hline & MAE & 4.33 & 3.4 & 18.29 & 11.52 & 10.38 & 12.16 & 11.61 \\
\hline & MARE [\%] & 12.28 & 12.65 & 51.53 & 34.64 & 29.47 & 35.61 & 30.20 \\
\hline
\end{tabular}




\section{Vertical ionization potentials}

TABLE S10: Vertical ionization potential (in eV) corresponding to various methods calculated as an energy difference between the neutral and the ionic species $(V I P=E(N)-E(N-1))$. The last lines report the mean absolute error (MAE) and mean absolute relative error (MARE) calculated with respect $\operatorname{CSSD}(\mathrm{T})$ data. The computational setup, namely basis sets and geometries in case of molecules, is identical as in J. Comput. Chem. 37 (2016), 2081. ${ }^{a}$ ) with TPSS-GL2

\begin{tabular}{|c|c|c|c|c|c|c|c|c|}
\hline & \multirow[b]{2}{*}{$\operatorname{CCSD}(\mathrm{T})$} & \multirow[b]{2}{*}{ MP2 } & \multirow[b]{2}{*}{ OEP2-sc } & \multicolumn{5}{|c|}{ @OEPx } \\
\hline & & & & GL2 & ISI & mISI & $\mathrm{mISI}^{a}$ & ACSC \\
\hline $\mathrm{Ar}$ & -15.63 & -15.77 & -15.75 & -16.31 & -16.01 & -15.94 & -16.06 & -15.76 \\
\hline $\mathrm{Be}$ & -9.31 & -8.88 & -8.88 & -10.01 & -9.51 & -9.49 & -9.14 & -8.82 \\
\hline $\mathrm{C}_{2} \mathrm{H}_{2}$ & -11.43 & -11.6 & -11.64 & -12.94 & -11.94 & -11.77 & -11.08 & -10.91 \\
\hline $\mathrm{C}_{2} \mathrm{H}_{4}$ & -10.63 & -10.65 & -10.7 & -11.94 & -11.01 & -10.85 & -10.11 & -9.95 \\
\hline $\mathrm{C}_{2} \mathrm{H}_{6}$ & -13.01 & -13.11 & -12.67 & -12.74 & -12.69 & -12.68 & -13.23 & -13.19 \\
\hline $\mathrm{CH}_{2} \mathrm{CF}_{2}$ & -10.61 & -10.67 & -10.5 & -11.3 & -10.83 & -10.74 & -10.77 & -10.58 \\
\hline $\mathrm{CH}_{3} \mathrm{CN}$ & -10.75 & -11.33 & -10.76 & -12.47 & -11.53 & -11.36 & -10.94 & -10.72 \\
\hline $\mathrm{CH}_{4}$ & -14.37 & -14.4 & -14.41 & -14.82 & -14.46 & -14.4 & -14.52 & -14.41 \\
\hline $\mathrm{CHF}_{3}$ & -14.59 & -14.61 & -14.23 & -15.87 & -15.93 & -15.94 & -16.95 & -16.85 \\
\hline $\mathrm{Cl}_{2}$ & -11.45 & -11.5 & -11.37 & -11.06 & -11.09 & -11.1 & -12.54 & -12.29 \\
\hline $\mathrm{CO}_{2}$ & -13.7 & -14.63 & -13.85 & -15.51 & -14.63 & -14.46 & -14.03 & -13.79 \\
\hline $\mathrm{CO}$ & -13.94 & -14.31 & -13.71 & -14.88 & -14.35 & -14.24 & -14.32 & -14.21 \\
\hline $\mathrm{CS}$ & -11.27 & -11.63 & -11.19 & -14.52 & -13.73 & -13.58 & -11.64 & -11.23 \\
\hline $\mathrm{FCN}$ & -13.65 & -13.85 & -13.72 & -16.33 & -15.32 & -15.13 & -14.45 & -14.29 \\
\hline $\mathrm{H}_{2} \mathrm{CO}$ & -10.83 & -11.26 & -10.84 & -12.2 & -11.41 & -11.27 & -10.75 & -10.59 \\
\hline $\mathrm{H}_{2} \mathrm{CS}$ & -9.29 & -9.57 & -9.28 & -10.73 & -10.27 & -10.19 & -9.48 & -9.16 \\
\hline $\mathrm{H}_{2} \mathrm{O}$ & -12.5 & -12.69 & -12.7 & -13.6 & -12.86 & -12.73 & -12.16 & -12 \\
\hline $\mathrm{HCCF}$ & -11.5 & -11.54 & -11.44 & -12.49 & -11.8 & -11.67 & -11.41 & -11.2 \\
\hline $\mathrm{HCl}$ & -12.59 & -12.67 & -12.66 & -13.27 & -13.07 & -13.03 & -12.97 & -12.65 \\
\hline $\mathrm{HCN}$ & -13.9 & -14.72 & -14.09 & -16.44 & -15.1 & -14.86 & -13.81 & -13.66 \\
\hline $\mathrm{He}$ & -24.48 & -24.54 & -24.41 & -24.74 & -24.51 & -24.54 & -24.49 & -24.34 \\
\hline $\mathrm{He}_{2}$ & -24.48 & -24.54 & -24.52 & -24.7 & -24.52 & -24.54 & -24.74 & -24.8 \\
\hline $\mathrm{HF}$ & -15.96 & -16.18 & -16.2 & -17.05 & -16.38 & -16.26 & -15.57 & -15.41 \\
\hline $\mathrm{Mg}$ & -7.57 & -7.32 & -7.33 & -8.11 & -7.97 & -7.94 & -7.44 & -7.22 \\
\hline $\mathrm{N}_{2}$ & -15.51 & -15.34 & -15.06 & -13.41 & -14.59 & -14.76 & -16.66 & -16.64 \\
\hline $\mathrm{NCCN}$ & -13.51 & -14.85 & -13.77 & -12.62 & -13.89 & -14.08 & -16.52 & -16.49 \\
\hline $\mathrm{Ne}_{2}$ & -21.34 & -21.42 & -20.72 & -22.37 & -21.79 & -21.67 & -21.01 & -20.86 \\
\hline $\mathrm{Ne}$ & -21.47 & -21.67 & -21.69 & -22.39 & -21.83 & -21.72 & -21.04 & -20.88 \\
\hline $\mathrm{NH}_{3}$ & -10.78 & -10.86 & -10.88 & -11.69 & -11.01 & -10.9 & -10.52 & -10.39 \\
\hline OCS & -11.18 & -11.75 & -11.27 & -12.6 & -12.1 & -12 & -11.69 & -11.36 \\
\hline $\mathrm{P}_{2}$ & -10.66 & -10.68 & -10.55 & -10.43 & -10.4 & -10.39 & -11.41 & -11.06 \\
\hline \multirow[t]{3}{*}{$\mathrm{SiH}_{4}$} & -12.78 & -12.82 & -12.72 & -13.13 & -13 & -12.98 & -12.99 & -12.88 \\
\hline & MAE & 0.24 & 0.17 & 1.17 & 0.61 & 0.52 & 0.42 & 0.39 \\
\hline & MARE [\%] & 2.37 & 1.21 & 9.03 & 4.90 & 4.26 & 3.83 & 3.55 \\
\hline
\end{tabular}




\section{$5 \quad$ Binding energies}

TABLE S11: Binding energies (in $\mathrm{kcal} / \mathrm{mol}$ ) corresponding to various methods. The last lines report the mean absolute error (MAE) and mean absolute relative error (MARE) calculated with respect CCSD(T) data. All calculation have been performed with fully uncontacted cc-pVTZ basis set. ${ }^{a}$ ) with TPSS-GL2

\begin{tabular}{|c|c|c|c|c|c|c|c|c|}
\hline & \multirow[b]{2}{*}{$\operatorname{CCSD}(\mathrm{T})$} & \multirow[b]{2}{*}{ MP2 } & \multirow[b]{2}{*}{ OEP2-sc } & \multicolumn{5}{|c|}{ @OEPx } \\
\hline & & & & GL2 & ISI & mISI & $\mathrm{mISI}^{a}$ & ACSC \\
\hline \multicolumn{9}{|c|}{ WI } \\
\hline $\mathrm{He}-\mathrm{Ne}$ & -0.04 & -0.03 & -0.03 & 0.90 & 0.61 & 0.62 & 0.32 & 0.02 \\
\hline $\mathrm{He}-\mathrm{Ar}$ & -0.06 & -0.06 & -0.06 & 0.83 & 0.72 & 0.72 & 0.51 & 0.05 \\
\hline $\mathrm{Ne}-\mathrm{Ne}$ & -0.08 & -0.06 & -0.07 & -0.10 & -0.12 & -0.05 & 0.03 & 0.04 \\
\hline $\mathrm{Ne}-\mathrm{Ar}$ & -0.13 & -0.12 & -0.14 & -0.23 & -0.21 & -0.16 & 0.64 & 0.08 \\
\hline $\mathrm{CH}_{4}-\mathrm{Ne}$ & -0.22 & -0.18 & -0.21 & -0.32 & -0.31 & -0.17 & -4.99 & 0.05 \\
\hline $\mathrm{C}_{6} \mathrm{H}_{6}-\mathrm{Ne}$ & -0.45 & -0.61 & -0.71 & -1.26 & -0.97 & -0.55 & -6.78 & 0.18 \\
\hline $\mathrm{CH}_{4}-\mathrm{CH}_{4}$ & -0.59 & -0.56 & -0.65 & -1.25 & -0.97 & -0.53 & 0.22 & 0.24 \\
\hline \multicolumn{9}{|c|}{ HB6 } \\
\hline $\mathrm{HF}-\mathrm{HF}$ & -4.67 & -4.59 & -4.74 & -4.17 & -4.46 & -4.15 & -4.74 & -4.80 \\
\hline $\mathrm{H}_{2} \mathrm{O}-\mathrm{H}_{2} \mathrm{O}$ & -5.11 & -5.10 & -5.34 & -5.15 & -5.19 & -4.71 & -4.93 & -5.04 \\
\hline $\mathrm{NH}_{3}-\mathrm{H}_{2} \mathrm{O}$ & -6.57 & -6.66 & -7.00 & -7.13 & -6.86 & -6.26 & -6.73 & -6.49 \\
\hline $\mathrm{HF}-\mathrm{HCN}$ & -7.58 & -7.86 & -8.20 & -7.76 & -7.77 & -7.20 & -8.35 & -7.73 \\
\hline$\left(\mathrm{HCONH}_{2}\right)_{2}$ & -15.27 & -15.83 & -15.36 & -14.91 & -15.36 & -14.39 & -16.30 & -16.77 \\
\hline$(\mathrm{HCOOH})_{2}$ & -18.32 & -18.18 & -18.16 & -15.50 & -16.85 & -16.01 & -19.98 & -20.51 \\
\hline \multicolumn{9}{|c|}{ DI6 } \\
\hline $\mathrm{HCl}-\mathrm{HCl}$ & -2.26 & -2.56 & -2.71 & -3.74 & -3.3 & -3.15 & -1.32 & -1.28 \\
\hline $\mathrm{H}_{2} \mathrm{~S}-\mathrm{HCl}$ & -3.67 & -4.19 & -4.41 & -5.83 & -5.19 & -4.98 & -3 & -2.8 \\
\hline $\mathrm{CH}_{3} \mathrm{Cl}-\mathrm{HCl}$ & -3.98 & -4.49 & -4.81 & -6.59 & -5.67 & -5.36 & -3.6 & -2.5 \\
\hline $\mathrm{CH}_{3} \mathrm{SH}-\mathrm{HCN}$ & -3.96 & -4.25 & -4.52 & -5.46 & -4.71 & -4.29 & -2.63 & -3.28 \\
\hline $\mathrm{CH}_{3} \mathrm{SH}-\mathrm{HCl}$ & -5.34 & -6.13 & -6.56 & -8.84 & -7.61 & -7.22 & -5.79 & -3.99 \\
\hline \multicolumn{9}{|c|}{ DHB } \\
\hline $\mathrm{AlH}-\mathrm{HF}$ & -6.56 & -6.85 & -7.31 & -7.71 & -7.05 & -6.77 & -5.11 & -4.98 \\
\hline $\mathrm{LiH}-\mathrm{HCl}$ & -11.8 & -13.28 & -13.53 & -14.93 & -13.9 & -13.6 & -11.92 & -12.41 \\
\hline LiH-HF & -14.1 & -14.74 & -15.22 & -14.75 & -14.37 & -13.86 & -14.12 & -14.66 \\
\hline $\mathrm{MgH}_{2}-\mathrm{HCl}$ & -5.08 & -5.72 & -5.93 & -7.45 & -6.7 & -6.49 & -3.78 & -3.99 \\
\hline $\mathrm{MgH}_{2}-\mathrm{HF}$ & -7.38 & -7.66 & -8.05 & -8.21 & -7.66 & -7.39 & -6.66 & -6.58 \\
\hline $\mathrm{BeH}_{2}-\mathrm{Hcl}$ & -2.44 & -2.7 & -2.81 & -3.88 & -3.33 & -3.15 & -1.35 & -1.43 \\
\hline $\mathrm{BeH}_{2}-\mathrm{HF}$ & -3.66 & -3.72 & -3.96 & -4.2 & -3.85 & -3.45 & -2.82 & -2.94 \\
\hline \multicolumn{9}{|c|}{ CT7 } \\
\hline $\mathrm{C}_{2} \mathrm{H}_{4}-\mathrm{F}_{2}$ & -1.19 & -1.6 & -2.05 & -3.02 & -1.93 & -1.34 & -4.79 & 0.43 \\
\hline $\mathrm{NF}_{3}-\mathrm{HNC}$ & -1.85 & -1.99 & -2.46 & -3.6 & -2.57 & -2.01 & -1.42 & -0.33 \\
\hline $\mathrm{C}_{2} \mathrm{H}_{4}-\mathrm{Cl}_{2}$ & -2.6 & -4.14 & -5.09 & -8.96 & -6.73 & -6.1 & -8.02 & 0.11 \\
\hline $\mathrm{NH}_{3}-\mathrm{F}_{2}$ & -1.86 & -2.06 & -2.91 & -3.1 & -2.23 & -1.66 & -3.07 & -0.21 \\
\hline $\mathrm{NF}_{3}-\mathrm{ClF}$ & -2.03 & -2.44 & -3.43 & -4.96 & -3.54 & -2.95 & 0.5 & 0.18 \\
\hline $\mathrm{NF}_{3}-\mathrm{HF}$ & -2.26 & -2.33 & -2.75 & -3.62 & -2.77 & -2.25 & -0.97 & -0.9 \\
\hline $\mathrm{C}_{2} \mathrm{H}_{2}-\mathrm{ClF}$ & -3.85 & -5.04 & -5.95 & -7.73 & -5.88 & -5.18 & -6.52 & -2.18 \\
\hline $\mathrm{HCN}-\mathrm{ClF}$ & -4.87 & -5.8 & -6.89 & -7.93 & -6.54 & -5.95 & -5.33 & -3.3 \\
\hline $\mathrm{NH}_{3}-\mathrm{Cl}_{2}$ & -5.13 & -5.87 & -7.18 & -8.75 & -7.47 & -7.07 & -7.63 & -3.33 \\
\hline $\mathrm{H}_{2} \mathrm{O}-\mathrm{ClF}$ & -5.22 & -5.69 & -6.73 & -6.3 & -5.68 & -5.28 & -5.47 & -4.57 \\
\hline \multirow[t]{3}{*}{$\mathrm{NH}_{3}-\mathrm{ClF}$} & -2.03 & -2.44 & -3.43 & -4.96 & -7.7 & -9.33 & 0.50 & 0.18 \\
\hline & MAE & 0.38 & 0.74 & 1.63 & 1.05 & 0.91 & 1.37 & 1.03 \\
\hline & MARE[\%] & 12.58 & 24.28 & 154.22 & 113.00 & 103.06 & 195.24 & 58.49 \\
\hline
\end{tabular}




\section{GL2 correlation energies}

TABLE S12: The GL2 correlation energies obtained from exact and various semi-local energy expression (in mHa) calculated on top of self-consistent OEPx otbitalas and orbital energies in fully uncontacted cc-pVTZ basis set. The last lines report the mean absolute error (MAE) and mean absolute relative error (MARE) calculated with respect exact GL2 data.

\begin{tabular}{|c|c|c|c|c|}
\hline & GL2 & PBE-GL2 & TPSS-GL2 & revTPSS-GL2 \\
\hline $\mathrm{He}$ & -44.363 & -46.337 & -47.903 & -51.808 \\
\hline $\mathrm{Li}$ & -39.994 & -57.796 & -56.165 & -60.445 \\
\hline $\mathrm{Be}$ & -113.802 & -113.424 & -112.781 & -122.476 \\
\hline B & -134.885 & -140.928 & -137.370 & -147.979 \\
\hline $\mathrm{C}$ & -161.406 & -176.444 & -174.906 & -186.062 \\
\hline $\mathrm{N}$ & -191.277 & -217.471 & -219.668 & -231.197 \\
\hline $\mathrm{O}$ & -254.493 & -281.078 & -275.198 & -288.047 \\
\hline $\mathrm{F}$ & -326.869 & -346.140 & -341.461 & -355.356 \\
\hline $\mathrm{Ne}$ & -405.145 & -412.358 & -414.265 & -429.077 \\
\hline $\mathrm{BeH}$ & -97.580 & -124.940 & -119.303 & -129.022 \\
\hline $\mathrm{OH}$ & -340.946 & -349.456 & -342.201 & -357.811 \\
\hline $\mathrm{NH}_{2}$ & -327.428 & -357.601 & -347.322 & -364.718 \\
\hline $\mathrm{NH}$ & -254.288 & -286.955 & -277.722 & -292.113 \\
\hline $\mathrm{NO}$ & -723.276 & -627.878 & -619.234 & -645.573 \\
\hline $\mathrm{PH}_{2}$ & -422.856 & -746.632 & -735.950 & -764.970 \\
\hline $\mathrm{O}_{2}$ & -770.850 & -666.881 & -657.954 & -684.674 \\
\hline $\mathrm{S}_{2}$ & -918.035 & -1515.626 & -1501.791 & -1552.064 \\
\hline $\mathrm{SiH}_{2}$ & -347.422 & -654.585 & -643.779 & -670.087 \\
\hline $\mathrm{C}_{2} \mathrm{H}_{5}$ & -601.636 & -738.081 & -717.140 & -753.425 \\
\hline $\mathrm{CH}_{2}$ & -226.830 & -289.601 & -277.095 & -292.735 \\
\hline $\mathrm{CH}_{3}$ & -287.709 & -359.285 & -346.147 & -365.085 \\
\hline $\mathrm{CN}$ & -541.471 & -511.417 & -500.899 & -524.626 \\
\hline $\mathrm{COH}$ & -670.735 & -619.500 & -610.582 & -638.332 \\
\hline $\mathrm{CH}_{4}$ & -348.835 & -424.613 & -417.151 & -439.289 \\
\hline $\mathrm{LiF}$ & -487.876 & -451.807 & -453.721 & -473.896 \\
\hline $\mathrm{Li}_{2}$ & -137.012 & -164.787 & -162.619 & -177.591 \\
\hline $\mathrm{F}_{2}$ & -875.886 & -744.616 & -744.887 & -773.967 \\
\hline $\mathrm{CO}$ & -659.650 & -571.305 & -568.931 & -594.734 \\
\hline $\mathrm{CH}_{2}$ & -278.421 & -300.300 & -296.480 & -313.730 \\
\hline $\mathrm{H}_{2}$ & -47.537 & -57.984 & -53.775 & -58.555 \\
\hline CS & -781.015 & -1000.806 & -995.643 & -1033.483 \\
\hline $\mathrm{LiH}$ & -86.328 & -100.313 & -100.918 & -109.990 \\
\hline $\mathrm{N}_{2}$ & -708.486 & -595.291 & -592.501 & -618.479 \\
\hline $\mathrm{P}_{2}$ & -910.610 & -1403.130 & -1396.442 & -1445.947 \\
\hline $\mathrm{NaCl}$ & -729.401 & -1265.590 & -1264.610 & -1307.531 \\
\hline $\mathrm{H}_{2} \mathrm{O}$ & -433.049 & -418.419 & -416.233 & -434.688 \\
\hline & MAE & 105.03 & 101.89 & 110.28 \\
\hline & MARE [\%] & 21.99 & 15.45 & 18.87 \\
\hline
\end{tabular}




\section{Binding energies on top of HF orbitals}

TABLE S13: Binding energies (in kcal/mol) from MP2, ISI and mISI methods calculated on top of HF orbitals. The last lines report the mean absolute error (MAE) and mean absolute relative error (MARE) calculated with respect CCSD(T) data. All calculation have been performed with fully uncontacted cc-pVTZ basis set.

\begin{tabular}{|c|c|c|c|}
\hline & MP2 & ISI & mISI \\
\hline & WI & & \\
\hline $\mathrm{He}-\mathrm{Ne}$ & -0.03 & -0.05 & -0.02 \\
\hline $\mathrm{He}-\mathrm{Ar}$ & -0.06 & -0.05 & -0.04 \\
\hline $\mathrm{Ne}-\mathrm{Ne}$ & -0.06 & -0.08 & -0.04 \\
\hline $\mathrm{Ne}-\mathrm{Ar}$ & -0.12 & -0.11 & -0.09 \\
\hline $\mathrm{CH}_{4}-\mathrm{Ne}$ & -0.18 & -0.19 & -0.12 \\
\hline $\mathrm{C}_{6} \mathrm{H}_{6}-\mathrm{Ne}$ & -0.61 & -0.54 & -0.34 \\
\hline $\mathrm{CH}_{4}-\mathrm{CH}_{4}$ & -0.56 & -0.49 & -0.28 \\
\hline MAE & $\begin{array}{c}0.04 \\
\text { HB6 }\end{array}$ & 0.04 & 0.09 \\
\hline $\mathrm{NH}_{3}-\mathrm{NH}_{3}$ & -3.25 & -3.13 & -2.87 \\
\hline $\mathrm{HF}-\mathrm{HF}$ & -4.59 & -4.68 & -4.51 \\
\hline $\mathrm{H}_{2} \mathrm{O}-\mathrm{H}_{2} \mathrm{O}$ & -5.10 & -5.10 & -4.85 \\
\hline $\mathrm{NH}_{3}-\mathrm{H}_{2} \mathrm{O}$ & -6.66 & -6.54 & -6.24 \\
\hline HF-HCN & -7.86 & -7.83 & -7.54 \\
\hline$\left(\mathrm{HCONH}_{2}\right)_{2}$ & -15.83 & -15.80 & -15.27 \\
\hline$(\mathrm{HCOOH})_{2}$ & -18.18 & -18.35 & -17.84 \\
\hline MAE & $\begin{array}{c}0.17 \\
\text { DI6 }\end{array}$ & 0.14 & 0.23 \\
\hline $\mathrm{H}_{2} \mathrm{~S}-\mathrm{H}_{2} \mathrm{~S}$ & -2.15 & -1.98 & -1.92 \\
\hline Hcl-Hcl & -2.56 & -2.39 & -2.33 \\
\hline $\mathrm{H}_{2} \mathrm{~S}-\mathrm{Hcl}$ & -4.19 & -3.94 & -3.86 \\
\hline $\mathrm{CH}_{3} \mathrm{Cl}-\mathrm{Hcl}$ & -4.49 & -4.13 & -4.01 \\
\hline $\mathrm{CH}_{3} \mathrm{SH}-\mathrm{HCN}$ & -4.25 & -3.98 & -3.81 \\
\hline $\mathrm{CH}_{3} \mathrm{SH}-\mathrm{Hcl}$ & -6.13 & -5.67 & -5.52 \\
\hline MAE & $\begin{array}{l}0.45 \\
\text { DHB }\end{array}$ & 0.17 & 0.11 \\
\hline $\mathrm{AlH}-\mathrm{HCl}$ & -5.26 & -4.92 & -4.83 \\
\hline AlH-HF & -6.85 & -6.54 & -6.41 \\
\hline $\mathrm{LiH}-\mathrm{HCl}$ & -13.28 & -12.78 & -12.65 \\
\hline LiH-HF & -14.74 & -14.44 & -14.16 \\
\hline $\mathrm{MgH}_{2}-\mathrm{HCl}$ & -5.72 & -5.4 & -5.31 \\
\hline $\mathrm{MgH}_{2}-\mathrm{HF}$ & -7.66 & -7.38 & -7.24 \\
\hline $\mathrm{BeH}_{2}-\mathrm{HCl}$ & -2.7 & -2.49 & -2.42 \\
\hline $\mathrm{BeH}_{2}-\mathrm{HF}$ & -3.72 & -3.59 & -3.39 \\
\hline MAE & $\begin{array}{c}0.54 \\
\text { CT7 }\end{array}$ & 0.27 & 0.25 \\
\hline $\mathrm{NF}_{3}-\mathrm{HCN}$ & -1.24 & -1.2 & -1.00 \\
\hline $\mathrm{C}_{2} \mathrm{H}_{4}-\mathrm{F}_{2}$ & -1.6 & -1.29 & -1.03 \\
\hline $\mathrm{NF}_{3}-\mathrm{HNC}$ & -1.99 & -1.68 & -1.44 \\
\hline $\mathrm{C}_{2} \mathrm{H}_{4}-\mathrm{Cl}_{2}$ & -4.14 & -3.43 & -3.22 \\
\hline $\mathrm{NH}_{3}-\mathrm{F}_{2}$ & -2.06 & -1.77 & -1.51 \\
\hline $\mathrm{NF}_{3}-\mathrm{ClF}$ & -2.44 & -2.11 & -1.88 \\
\hline $\mathrm{NF}_{3}-\mathrm{HF}$ & -2.33 & -2.04 & -1.80 \\
\hline $\mathrm{C}_{2} \mathrm{H}_{2}-\mathrm{ClF}$ & -5.04 & -4.47 & -4.21 \\
\hline $\mathrm{HCN}-\mathrm{ClF}$ & -5.8 & -5.39 & -5.16 \\
\hline $\mathrm{NH}_{3}-\mathrm{Cl}_{2}$ & -5.87 & -5.44 & -5.29 \\
\hline $\mathrm{H}_{2} \mathrm{O}-\mathrm{ClF}$ & -5.69 & -5.45 & -5.27 \\
\hline $\mathrm{NH}_{3}-\mathrm{ClF}$ & -2.44 & -4.65 & -5.68 \\
\hline MAE & 0.55 & 0.48 & 0.57 \\
\hline MAE & 0.38 & 0.25 & 0.29 \\
\hline MARE[\%] & 12.58 & 10.26 & 17.11 \\
\hline
\end{tabular}

